\title{
Emprendimiento e innovación social: Respuesta colectiva frente a viejas y nuevas demandas sociales en América Latina
}

\author{
Social innovation and entrepreneurship: Collective response to old and new social demands \\ in Latin America
}

MONTENEGRO, Josué C. ${ }^{1}$

\section{Resumen}

Este artículo discute los determinantes de la inequidad en América Latina, entre los cuales se encuentra la falta de financiamiento, infraestructura y educación, y se centra en analizar cómo frente a esto, el emprendimiento y la innovación social han surgido como respuesta por parte de los mismos colectivos excluidos, últimamente estructurando un nuevo modelo de desarrollo económico y social caracterizado por la construcción de capacidades locales, transformación institucional, reconfiguración de recursos, e impulso a los objetivos de desarrollo sostenible.

Palabras Clave: emprendimiento social, innovación social, inequidad, desarrollo

\begin{abstract}
:
This article discusses the determinants of inequity in Latin America, among which some are the lack of financing, infrastructure, and education, and focuses on analyzing how, in the face of this, socially excluded groups have used entrepreneurship and social innovation to structure a new economic and social development model. This model is characterized by the construction of local capacities, institutional transformation, reconfiguration of resources, and promotion of sustainable development objectives.

Keywords: social entrepreneurship, social innovation, inequality, development
\end{abstract}

\section{Introducción}

Ante el inicio de la segunda década del siglo XXI, la sociedad latinoamericana se enfrenta a desafíos nuevos y antiguos que tienen el potencial de limitar el desarrollo social de sus pueblos y la inclusión de la región en los mercados internacionales. Los altos niveles de inequidad que por largo tiempo han caracterizado a América Latina, situándola tan solo por delante del África Subsahariana, siguen siendo una barrera inabordable a pesar de mejoras experimentadas en las últimas dos décadas (Gasparini, Cruces, \& Tornarolli, 2011; Lopez \& Perry, 2008). De hecho, si bien el índice de Gini se redujo desde un promedio ponderado de 0.550 a inicios del año 2000 a 0.465 para finales del 2019, aún hay factores de desigualdad estructurales que esconden una realidad compleja y que pueden impactar a la región a largo plazo (Székely \& Mendoza, 2017). En la misma línea y como respuesta a cambios propios de una nueva época, surgen desafíos relacionados a la institucionalidad 
democrática, consumo responsable, productividad de la mano de obra frente a la tecnología, y reducción de pobreza, entre otros que se ven plasmados en los objetivos de desarrollo sostenible (Naciones Unidas, 2020).

Resolver satisfactoriamente estos desafíos encuentra obstáculos en el sistema capitalista de mercado que privilegia las ganancias a corto plazo sobre resultados sistémicos a largo plazo. De hecho, en línea con la teoría económica clásica que sugiere que el interés propio de consumidores y productores lleva a un resultado eficiente de asignación de recursos y maximización de la utilidad (Smith, 2001), las empresas han optado por recurrir a restructuraciones, reducción de personal y reubicación a zonas con bajos costos productivos como estrategias para aumentar su eficiencia, enfrentar a la competencia y satisfacer la presión por resultados rápidos de sus accionistas (Porter \& Kramer, 2011). Dada la presencia de fallas institucionales, estas dinámicas han resultado también en externalidades que han extrapolado, por ejemplo, problemas ambientales. En otras palabras, pese a un panorama social con múltiples necesidades, las empresas han optado por un modelo tradicional de captura de valor en el que ha primado la generación de productos y servicios para aquellos que pueden pagar por ellos, obviando la alta demanda por parte de sectores que no cuentan con recursos financieros (Santos, 2012).

Ante esta situación, los gobiernos, fundaciones y ONGs han procurado asumir funciones redistributivas. Sin embargo, son pocos los resultados alcanzados debido a la limitación de sus fondos, desconocimiento, burocracia y afán de perpetuación (Yunus, 2008). De hecho, si bien es ideal que los gobiernos asuman funciones redistributivas y lidien con externalidades a través de regulación, impuestos y creación de mercados, muchas veces amplias necesidades locales pasan completamente desapercibidas, especialmente si estas están vinculadas con externalidades positivas y por ende con la sub-provisión o el sub-consumo de bienes (Rangan, Samii, \& Van Wassenhove, 2006). Esta situación es más acentuada cuando se habla de la provisión de bienes que no califican como públicos y cuya provisión impacta a grupos con escaso poder político, como minorías étnicas y poblaciones aisladas (Santos, 2012). En el mismo sentido, fundaciones y ONGs encuentran obstáculos para resolver los problemas que intentan abordar. De hecho, la misma dinámica de estas instituciones fundamentada en donaciones permanentes limita de gran manera las causas que pueden atacar y la escala a la que pueden llegar.

En el contexto anteriormente mencionado, el emprendimiento y la innovación social (E\&IS) surgen como mecanismos de reivindicación de las bases e instrumentos que dadas su características hibridas, en cuanto a combinación de valor social y financiero, permiten atender problemas sociales más profundos por un mayor tiempo. Se puede decir también que el E\&IS se caracterizan por crear valor en áreas donde tanto mercados como gobiernos fallan, al mismo tiempo que utilizan modelos comerciales para sostener sus operaciones y escalar (Santos, Pache, \& Birkholz, 2015). Similarmente, se ha visto en esta rama el surgimiento de nuevas propuestas tecnológicas, productos, procesos, colaboraciones y métricas de impacto que los aleja de patrones establecidos y de soluciones mercantiles comunes, llevando a lo que Joseph Schumpeter denominó como destrucción creativa (Hall \& Vredenburg, 2003). En este sentido, es posible ver que el E\&IS han tomado realce en cuanto a las posibilidades que ofrecen para superar problemas sociales como la pobreza, inequidad y falta de movilidad social, especialmente en economías emergentes donde se carece de recursos.

Con el objetivo de explicar el rol que el emprendimiento social y la innovación pueden tener en solucionar los desafíos antiguos y nuevos que enfrenta América Latina, así como el nuevo modelo que presentan frente al capitalismo clásico y la limitada acción gubernamental, este documento discutirá los determinantes de la inequidad en la región y posteriormente analizará al E\&IS, desde su conceptualización, aplicación y escala de acción, brindando una perspectiva clara acerca de su poder de trasformación social.

\section{Metodología}

El emprendimiento y la innovación social son dos temas muy entrelazados, cuyo análisis, entendimiento y debate dista de ser un tema resulto o completamente delineado. De hecho, mientras que algunos autores se han 
enfocado en explorar las oportunidades que estos dos fenómenos guardan para la creación de riqueza (Austin, Stevenson, \& Wei-Skillern, 2006; Dees, Anderson, \& Wei-Skillern, 2004), otros han explorado su aplicación práctica en la formulación de modelos de negocios (Dorado, 2006; Spear, 2006) y se han concentrado en definir y redefinir los objetivos prácticos alcanzables y la extensión de cada uno de estos conceptos (P. Dacin, Dacin, \& Matear, 2010; Zahra, Gedajlovic, Neubaum, \& Shulman, 2009). Como resultado, el entendimiento sobre los alcances e interrelación del emprendimiento e innovación social se han mantenidos difusos (T. Dacin, Dacin, \& Tracey, 2011).

En este escenario, y considerando el llamado a enfoques teóricos que amplíen el alcance del E\&IS de manera específica y lo aborden desde una perspectiva vinculada al emprendimiento institucional, movimientos sociales y desarrollo local (Mair \& Marti, 2006), la presente investigación tomará un carácter exploratorio y será sustentada en un enfoque inductivo que permita a través de observaciones particulares extraer conclusiones generales. De igual manera, y para asegurar un nivel alto de calidad en la información aquí utilizada, se siguió un proceso de revisión sistemática de literatura. Este proceso metodológico reduce sesgos posibles, permite administrar eficientemente gran cantidad de información y facilita el condensar los resultados obtenidos en los análisis (Fu, Kok, Dankbaar, Ligthart, \& van Riel, 2018; Salim, Ab Rahman, \& Abd Wahab, 2019).

Concretamente, la revisión sistemática de literatura fue organizada en cuatro fases. La primera de ellas comprendió la identificación de bases de datos y la búsqueda de información secundaria (i.e., artículos, reportes, libros) bajo parámetros de selección tales como tema, relevancia de palabras clave, y tiempo de publicación. Para esta búsqueda se utilizaron las bases de datos Google Scholar, Emerald y Scopus dado su amplio uso en investigación académica y rango de artículos científicos, libros, y reportes técnicos de organizaciones especializadas. Posteriormente, el material encontrado fue filtrado siguiendo para ello un criterio de inclusión y exclusión fundamentado en el alineamiento de la introducción, resumen y conclusiones con tópicos clave. Entre estos tópicos, algunos a mencionar son: educación, fallas institucionales, ecosistemas, emprendimiento local, empoderamiento ciudadano, valor social, movilidad social, desarrollo sostenible, construcción de capacidades y América Latina. De un total de 90 artículos y reportes pre-identificados, el proceso anteriormente mencionado permitió descartar 29, dejando en total 61 documentos para ser examinados posteriormente. Seguidamente, los documentos fueron sometidos a un análisis descriptivo y de contenido. En el análisis descriptivo, los documentos fueron agregados por categoría (i.e., temporalidad, metodología, área temática, alcance regional), lo que permitió ampliar la perspectiva del investigador sobre el alcance obtenido por estudios anteriores e identificar vacíos conceptuales a ser llenados. Finalmente, el análisis de contenido comprendió el estudio y revisión a profundidad de los documentos y categorías para identificar con ello conexiones posibles, fortalezas, debilidades y códigos de información para la construcción del análisis. Entre estos códigos, algunos encontrados fueron: crecimiento económico, capacidades, productividad, competitividad, tejido social, valor compartido, y creatividad ciudadana.

Empero a lo mencionado se reconocen limitaciones en este estudio. Específicamente, el hecho de que si bien el mismo se fundamentó en información secundaria procedente de amplias fuentes bibliográficas y se acoto a América Latina, este dista de ser completo. Especialmente beneficioso para posteriores investigaciones sería la utilización de información cuantitativa que permita la comparación de resultados y medición de impacto de iniciativas de emprendimiento social, en, por ejemplo, empoderamiento ciudadano, consolidación de instituciones y desarrollo humano. 


\section{Discusión}

\subsection{Determinantes de la Inequidad en América Latina}

Considerándose al índice de Gini como el principal indicador de los niveles de inequidad en la región, es posible observar una marcada mejora. De hecho, entre el periodo 2000 - 2012, la mayoría de países, a excepción de Guatemala, Costa Rica y Panamá, experimentaron una reducción en este indicador, con una disminución porcentual de más del $20 \%$ en el caso de República Dominicana, Paraguay, Uruguay y Argentina, y de entre $5 \%$ y $18 \%$ en el resto de países (Székely \& Mendoza, 2017). Sin embargo, la inequidad dista mucho de ser un problema del pasado en América Latina y continua respondiendo a dinámicas estructurales que no pueden ser solventadas con medidas de corte rápido o coyuntural. Es tal el caso que si bien los países han experimentado un crecimiento económico constante, según la Comisión Económica para América Latina y el Caribe (2017), 30\% de latinoamericanos aún viven en la pobreza y $10 \%$ sufre de condiciones de pobreza extrema. $Y$ es que cuando la inequidad se encuentra tan marcada en el tejido social de un territorio, la elasticidad en la reducción de la pobreza es menor en valores absolutos, lo cual implica que los países requieren crecer más rápidamente y a una tasa mayor en comparación con otros que no presentan niveles tan altos de desigualdad (Lopez, 2006; Perry, Arias, López, Maloney, \& Servén, 2006). Conforme lo sugieren Lopez y Perry (2008), si el nivel de desigualdad en América Latina fuera similar al de Europa, la tasa de pobreza sería cercana a $12 \%$.

La alta inequidad responde a factores varios, entre los cuales, cabe especialmente mencionar a las diferencias que distintos segmentos sociales afrentan en cuanto a distribución de activos (especialmente educación), acceso financiero y a infraestructura, barreras laborales, y falencias en las instituciones gubernamentales (Banco Interamericano de Desarrollo, 2001). Esto termina determinando, en línea con la teoría de capacidades de Sen (2001), el que ciertos individuos se priven de la habilidad de desarrollarse de forma autónoma en la manera que consideren adecuada para ellos. En este contexto, por ejemplo, carencias en educación (e.g. baja calidad de la enseñanza, mala infraestructura pública, problemas de salud y cognitivos, y limitado acceso al crédito) predicen la generación de ingresos y movilidad social posible.

En efecto, las diferencias en cuanto a logros educativos entre los quintiles más altos y bajos en la región resultan marcadas. Como lo menciona la Organización de las Naciones Unidas para la Educación, la Ciencia y la Cultura (2014), las personas en el quintil más pobre (especialmente indígenas y afrodescendientes) tienen una probabilidad 7 veces más alta de no terminar su educación primaria en relación a aquellos en el quintil más rico. Situación que se explica debido a la convexidad presente en retornos a la educación (i.e. mayor retorno a la educación mientras mayor es la misma) y al costo de oportunidad que enfrentan las familias pobres al enviar a sus hijos a un sistema educativo por periodos prolongados. En otras palabras, existe un círculo vicioso que favorece la perpetuación de inequidades. Mientras los quintiles más ricos y la clase media incursionan en el emprendimiento comercial, inversión e innovación apalancados por varios factores, especialmente su nivel educativo, los más pobres y marginados deben optar por la informalidad, subempleo, y emprendimiento de baja productividad (Ardagna \& Lusardi, 2008; Doepke \& Zilibotti, 2005). En esta línea, cuando un escenario con alta inequidad y baja movilidad persiste, pocos son los incentivos que las personas tienen para trabajar y tomar riesgos (Lopez \& Perry, 2008)2.

Desde un punto de vista económico, la inequidad también afecta a la productividad y competitividad del aparato productivo a través de diferentes canales. De hecho, a través del efecto que la desigualdad ejerce sobre la

\footnotetext{
${ }^{2}$ La baja movilidad social es especialmente relevante en Brasil, Ecuador y Guatemala, mientras que en países como Chile, Argentina, Uruguay, Perú y México existe mayor movilidad (Lopez \& Perry, 2008).
} 
sociedad, fenómenos como la criminalidad y la violencia empiezan a tener cada vez cabida ${ }^{3}$, ya que las personas se ven incentivadas a actuar por fuera de los mercados legales y problemas tales como alcoholismo, tráfico de drogas, conflictos armados y embarazo temprano crecen (Alesina \& Perotti, 1996; Banco Interamericano de Desarrollo, 2001). Esto se traduce en menos ingresos generados, bajo crecimiento poblacional, limitada adquisición de habilidades, y migración de mano de obra capacitada (Glaeser, Resseger, \& Tobio, 2009). Así mismo, la inequidad agudiza la volatilidad productiva ante la presencia de shocks económicos. Esto se da debido a que la falta de acceso a activos por parte de los quintiles pobres, información, tecnología, baja productividad del aparato productivo y limitada integración financiera limitan la acumulación de capital, y fuerzan un esquema económico frágil.

\subsection{El Emprendimiento y la Innovación Social como Mecanismos de Desarrollo}

Frente a un panorama de desigualdad en que el cerca de 4 billones de personas en el mundo - aquellas en la base de la pirámide y que ganan menos de dos dólares al día- son desatendidas o sub atendidas tanto por mercados formales y gobiernos, el E\&IS surgen como mecanismos de desarrollo local, cuyos procesos conjuntos y características propias pueden dar lugar a soluciones de carácter global (London \& Hart, 2011; Zahra, Rawhouser, Bhawe, Neubaum, \& Hayton, 2008). En líneas generales, mientras que enfoques tradicionales han relegado a poblaciones en vulnerabilidad a ser actores secundarios dentro de su propio progreso, la innovación y el emprendimiento social se han fundamentado en crear propuestas de valor que reconocen a las poblaciones locales como interpretes competentes de sus propias vidas, cuyo conocimiento y red de contactos les permite crear soluciones costo eficientes frente a problemas económicos, sociales y ambientales (Maclean, Harvey, \& Gordon, 2013; Mulgan, 2006; Núñez, 2010).

En esta línea, se reconoce el impacto que el E\&IS han tenido en áreas como el comercio justo, la generación de empleo temporal, reducción de la pobreza, manejo de residuos, educación e inclusión financiera (Alvord, Brown, \& Letts, 2004; Becker, Kunze, \& Vancea, 2017), especialmente en países en desarrollo, en donde los recursos son escasos y las instituciones a cargo de la política social corruptas o demasiado burocráticas para entender las necesidades locales, la extensión de las externalidades positivas y las estructuras de poder (Prahalad, 2006; Zahra et al., 2008). A través de la implementación de nuevos modelos de negocio, fundamentados en la generación de valor social por medio de la construcción de capacidades locales, formación de movimientos y diseminación de proyectos (Alvord et al., 2004), el E\&IS han contribuido a reformar estructuras sociales, arreglar el tejido social cuando este está fracturado, y atender necesidades que de otra forma habrían pasado desapercibidas. Como lo menciona Zahra, Gedajlovic, Neubaum, y Shulman (2009), el espectro de acción del E\&IS es amplio, ya que la generación de valor social trasciende espacios predefinidos, sustentándose sobre necesidades diversas de mayor índole que tan solo la producción de servicios para la captura de valor económico.

Es de esta forma que el E\&IS pueden surgir para atender necesidades específicas o incluso episódicas en su naturaleza pero también consolidar esquemas formales y soluciones escalables que permitan atender necesidades sociales más generales que no encuentran un espacio en mercados tradicionales. De igual manera, el componente de innovación social puede buscar transformar instituciones y crear reformas que viabilicen el desarrollo de comunidades y de grupos que anteriormente no contaban con una voz o presencia en los procesos de toma de decisiones. Algunos ejemplos de esto son visibles en las acciones realizadas por diversas organizaciones como BRAC o Grameen Group, que no solo han trabajado con comunidades desfavorecidas en brindarles capacitación y redes de contacto, sino también en reconfigurar recursos técnicos (e.g. créditos

\footnotetext{
${ }^{3}$ Según Fajnzylber, Lederman, y Loayza (2002), el incremento de un punto porcentual en el índice de desigualdad Gini lleva a un incremento en la tasa de criminalidad entre $1 \%$ y $4 \%$.
} 
bancarios), crear redes de emprendimiento, y fomentar cambios en las estructuras de poder (e.g. impulsando políticas de género, inclusión de grupos marginados).

En esta misma línea cabe mencionarse, que el E\&IS se caracterizan por construir propuestas de valor en conjunto con otros actores del sistema tales como corporaciones, instituciones de desarrollo, gobiernos, asociaciones, universidades, y ONGs (Nicholls, 2008). El trabajar con otros permite, en línea con lo que menciona la teoría de estructuración de Giddens $(1979,1984)$, profundizar el impacto de los cambios sociales objetivo, ya que el formar redes de apoyo facilita el acceso a recursos críticos, aumentando por ende la eficiencia y sostenibilidad de las operaciones (Calton, Werhane, Hartman, \& Bevan, 2013; Meyskens, Carsrud, \& Cardozo, 2010). A su vez, como lo argumentan Fukuyama (2001) y Estrin, Mickiewicz, y Stephan (2013), al crear estas alianzas, los actores del ecosistema de E\&IS consolidan redes blandas de capital social creando puentes entre grupos heterogéneos, fomentando un radio más amplio de solidaridad y confianza. Esto a su vez contribuye al mayor intercambio de información, reduce los costos de transacción y genera externalidades positivas, permitiendo el acceso de individuos a mayores oportunidades y recursos (i.e. movilidad social).

Así mismo, un elemento importante a destacar es que el E\&IS se distinguen, cuando van en conjunto, claramente de otras iniciativas sociales como programas de responsabilidad social corporativa, organizaciones sin fines de lucro y ONGs en que se busca generar modelos y programas que sean financieramente sostenibles, en donde la creación de valor social sea la prioridad pero también se compensen los costos económicos y sociales resultantes de las actividades (Zahra et al., 2009). En la misma línea, organizaciones resultantes de procesos de E\&IS, como las empresas sociales, aplican procesos de mercado, generan productos y servicios, cobran por ellos, generan ganancias $\mathrm{y}$ atraen inversionistas pero se distinguen fundamentalmente de las empresas comerciales tradicionales en que se atiende a aquellas áreas en donde existen derrames de valor más allá de las transacciones directas, se reinvierten las utilidades en la operación y perfeccionamiento del sistema, y se brinda soluciones a usuarios que tienen dificultades de acceso a mercados tradicionales o que no tienen capacidad o voluntad de pago (debido a desconocimiento) (Santos et al., 2015).

De hecho, mientras que en una transacción comercial tradicional, el valor esta dado únicamente por el producto intercambiado entre un vendedor y comprador, el E\&IS atiende áreas de impacto a través de variadas intervenciones, dentro de las cuales una puede ser el intercambio de bienes o servicios. Por ejemplificar, aunque los microcréditos, producto nacido del E\&IS, puedan pensarse a primera vista como un producto financiero per se, su poder va más allá y radica en permitir a grupos anteriormente marginados del sistema financiero (i.e. por falta de referencias, empleos informales, etc.) acceder a recursos y capacitación para llevar adelante actividades de emprendimiento a pequeña escala, logrando con ello un empoderamiento personal de los beneficiarios y propiciando la generación de excedentes que puedan ser invertidos en educación o salud.

En esta línea, es posible argumentar que el E\&IS proponen un nuevo modelo de desarrollo en el que la generación de valor económico está supeditada al progreso social de los sujetos y comunidades que forman parte del sistema. Este progreso a su vez se caracteriza por el fomento al desarrollo sostenible de los pueblos y se materializa en las líneas de acción planteadas en los objetivos de desarrollo del milenio (i.e. derechos humanos, reducción de la pobreza, educación, salud, empoderamiento y equidad, sostenibilidad ambiental) (Seelos \& Mair, 2005). A su vez, y como parte del sistema de mercado, se enfatiza particularmente la creación de habilidades y capacidades que aumenten la competitividad de las personas a través del incremento en la base de sus activos, así como mayor accesibilidad, aceptabilidad, asequibilidad y disponibilidad de información y tecnología que permitan conseguir incrementos en la productividad, mayor participación en el mercado y reducción de riesgos (Banco Interamericano de Desarrollo, 2001). En esta línea, se puede decir que, conforme lo mencionan Mongelli \& Rullani (2017), el modelo de desarrollo planteado por el E\&IS busca que aquellos que no han pertenecido a la clase creativa de la sociedad encuentren formas para desarrollar su creatividad, creen nuevos nichos, trabajos e 
industrias, generando dinámicas que ayuden a mitigar la polarización de las sociedades y contribuyan a difundir prosperidad.

\section{Gráfico 1}

Modelo de Desarrollo Fomentado por el Emprendimiento y la Innovación Social

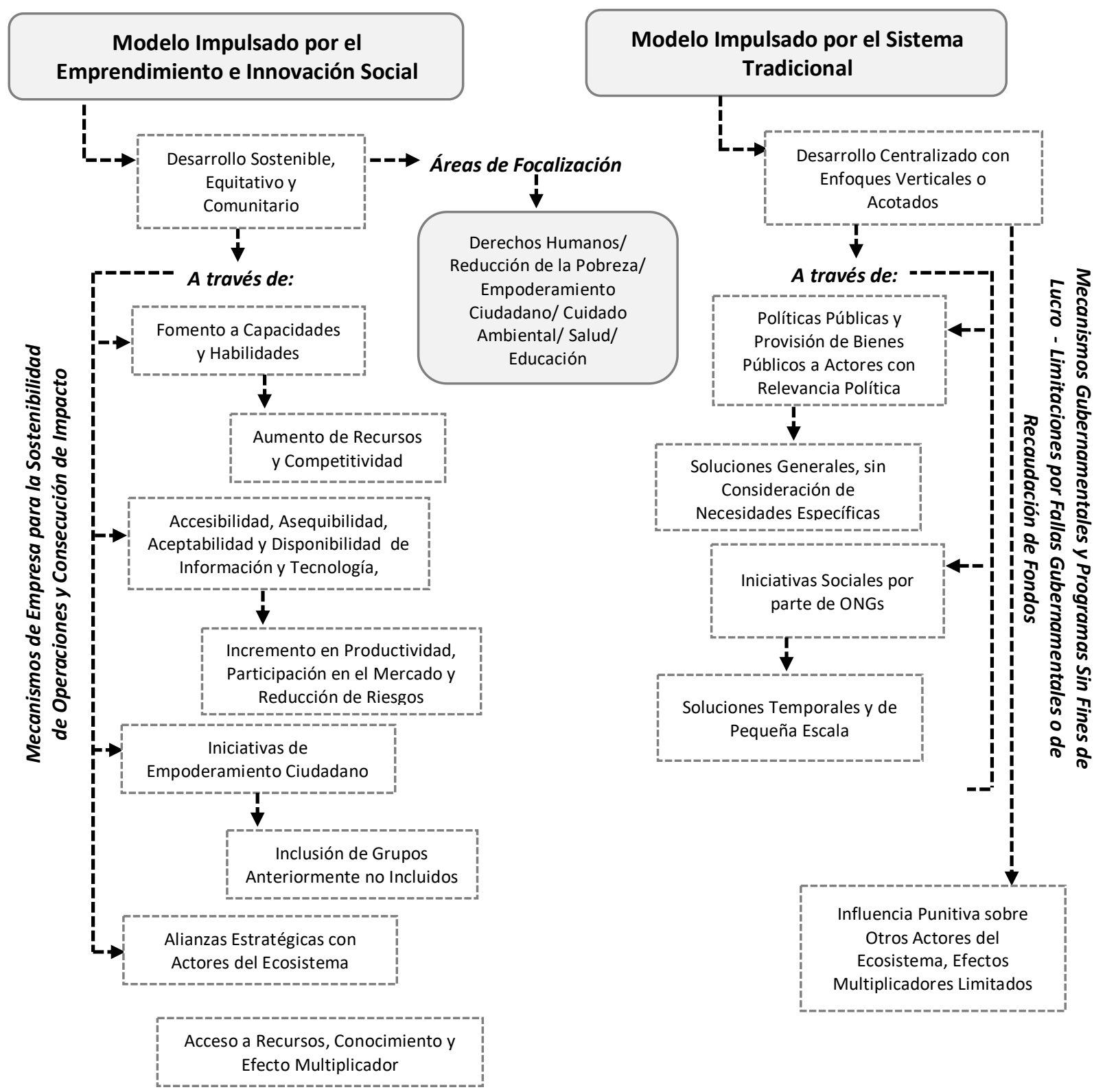

Elaboración: Autor

Fuentes: Mongelli \& Rullani (2017), Yunus et al., (2010), Rangan et al., (2006)

Por otro parte, es necesario reconocer que el modelo de desarrollo del E\&IS ha impactado en sectores de la economía tradicional a través de efectos multiplicadores, poniendo en marcha una amplia transformación del sistema económico e institucional. En específico, conforme lo mencionan Driver (2011) y Yunus, Moingeon, \& Lehmann-Ortega (2010), las empresas, por ejemplo, han empezado a cuestionar el rol que cumplen en la sociedad más allá de la simple provisión de bienes y servicios. Enfrentados a un contexto en que surgen organizaciones locales que satisfacen mejor las necesidades de las poblaciones y a un escenario en que los consumidores muestran su preocupación social a través de sus patrones de consumo, las corporaciones 
empiezan a reconocer que atender las necesidades sociales debe ser un aspecto central de su giro de negocio, yendo más allá de los programas de responsabilidad social corporativa. De hecho, según Porter \& Kramer (2011), la noción de valor compartido, que involucra aumentar la competitividad de una compañía mientras al mismo tiempo se mejoran las condiciones económicas y sociales de las comunidades en que esa empresa opera empieza a tomar fuerza. Lo anteriormente mencionado puede observarse en el Grafico 1, que presenta los ejes que estructuran el nuevo modelo de desarrollo, su impacto y como este se diferencia del modelo socio-económico actual.

\section{Conclusiones}

En América Latina, la inequidad y la desigualdad han permeado durante mucho tiempo las capas sociales de la población, contribuyendo a una segregación explicita de amplios segmentos de la sociedad, los cuales al verse desposeídos y limitados en su adquisición de capital físico y humano, debido a barreras institucionales y económicas, han resultado desfavorecidos y marginados por los procesos de desarrollo económico. En este contexto, incluso las acciones gubernamentales y de agentes del ámbito social tradicional, como ONGs y fundaciones, han resultado insuficientes. De hecho, los mismos elementos que ponen en juego la institucionalidad democrática de las naciones, como son la corrupción y la excesiva burocracia, han impedido a los gobiernos atender las amplias necesidades de la población, obviando la atención de externalidades positivas, a través de la provisión directa de bienes y servicios que cumplan con este fin o en su ausencia con el estímulo a otros actores que si pueden hacerlo. De forma similar, los agentes del ámbito social se han visto impedidos de expandir sus campos de acción debido a la frágil estructura que poseen en cuanto a sostenibilidad financiera, la misma que restringe los beneficiarios a los que pueden llegar y los problemas que pueden atacar.

Frente a este escenario, surge una alternativa en la forma del modelo de desarrollo que propone el E\&IS. En específico, lejos del esquema tradicional en que se le asigna a los grupos marginados y vulnerables un rol secundario y pasivo dentro de su propio progreso, el E\&IS construye nuevas propuestas de valor y modelos a partir de la acción directa de los propios beneficiarios, los cuales a través de su conocimiento local y redes de apoyo pueden crear, consolidar y escalar iniciativas que atiendan mejor sus necesidades. Así mismo, y como parte de un proceso transformador, este nuevo modelo de desarrollo va más allá de la provisión de bienes y servicios y se sustenta en la generación de valor por medio de la restructuración del tejido social, consolidando programas en donde se empodera y se da espacio de decisión a actores que no asumían este rol en el pasado (e.g. mujeres, ancianos). De igual manera, y como una propuesta que surge del mercado, el E\&IS formula un nuevo capitalismo, en el que el centro de atención se encuentra en las comunidades a las que se sirve y en el cual los mecanismos de empresa, como son la competencia, la inversión y la producción se orientan a generar sostenibilidad de operaciones y profundización de impacto.

En este marco, se puede argumentar que si el modelo de desarrollo del E\&IS es fortalecido, reconocido y apoyado, los determinantes de la inequidad en América Latina pueden ser contrarrestados. El tener mecanismos de desarrollo local, sostenibles y participativos aseguraría que al margen de las acciones implementadas por otros actores (e.g. gobiernos, ONGs), surjan iniciativas propias por parte de las comunidades que permitan combatir eficazmente la pobreza, la falta de acceso a infraestructura, la insuficiencia y baja calidad de centros de formación, además de los problemas sociales que vienen acarreados por estas falencias, como la criminalidad, la migración forzada y la violencia interpersonal. A su vez, la mayor inclusión se reflejaría en movilidad social y en la formación de actitudes conducentes a innovación e inversión (Castellani \& Lora, 2014; Doepke \& Zilibotti, 2005), fortaleciendo la creación de un sistema económico diferente. 


\section{Referencias Bibliográficas}

Alesina, A., \& Perotti, R. (1996). Income distribution, political instability, and investment. European Economic Review, 40(6), 1203-1228. https://doi.org/10.1016/0014-2921(95)00030-5

Alvord, S., Brown, D., \& Letts, C. (2004). Social Entrepreneurship and Societal Transformation: An Exploratory Study. The Journal of Applied Behavioral Science, 40(3), 260-282. https://doi.org/10.1177/0021886304266847

Ardagna, S., \& Lusardi, A. (2008). Explaining International Differences in Entrepreneurship: The Role of Individual Characteristics and Regulatory Constraints. NBER Working Paper Series, 14012. https://doi.org/10.3386/w14012

Austin, J., Stevenson, H., \& Wei-Skillern, J. (2006). Social and commercial entrepreneurship: same, different, or both? Entrepreneurship Theory and Practice, 30(1), 1-22.

Banco Interamericano de Desarrollo. (2001). Poverty and Inequality in Latin America and the Caribbean. Recuperado de https://publications.iadb.org/en/publication/poverty-and-inequality-latin-america-andcaribbean

Becker, S., Kunze, C., \& Vancea, M. (2017). Community energy and social entrepreneurship: Addressing purpose, organisation and embeddedness of renewable energy projects. Journal of Cleaner Production, 147, 25-36. https://doi.org/10.1016/j.jclepro.2017.01.048

Calton, J., Werhane, P., Hartman, L., \& Bevan, D. (2013). Building Partnerships to Create Social and Economic Value at the Base of the Global Development Pyramid. Journal of Business Ethics, 117(4), 721-733. https://doi.org/10.1007/s10551-013-1716-0

Castellani, F., \& Lora, E. (2014). Is entrepreneurship a channel of social mobility in Latin America? Latin American Journal of Economics, 51(2), 179-194. https://doi.org/10.7764/LAJE.51.2.179

Comisión Económica para América Latina y el Caribe. (2017). La pobreza aumentó en 2016 en América Latina y alcanzó al 30,7\% de su población, porcentaje que se mantendría estable en 2017 | Comunicado de prensa | Comisión Económica para América Latina y el Caribe. Retrieved April 18, 2020, from https://www.cepal.org/es/comunicados/la-pobreza-aumento-2016-america-latina-alcanzo-al-307-supoblacion-porcentaje-que-se

Dacanay, M. L. M. (2004). Creating a space in the market: social enterprise stories in Asia. Asian Institute of Management.

Dacin, P., Dacin, M., \& Matear, M. (2010). Social entrepreneurship: Why we don't need a new theory and how we move forward from here. Academy of Management Perspectives, 24(3), 37-57.

Dacin, T., Dacin, P., \& Tracey, P. (2011). Social entrepreneurship: A critique and future directions. Organization Science, 22(5), 1203-1213.

Dees, J. G., Anderson, B. B., \& Wei-Skillern, J. (2004). Scaling social impact. Stanford Social Innovation Review, 1(4), 24-32.

Doepke, M., \& Zilibotti, F. (2005). Social Class and the Spirit of Capitalism. Journal of the European Economic Association, 3(2/3), 516-524. https://doi.org/10.1162/1542476054473215

Dorado, S. (2006). Social entrepreneurial ventures: different values so different process of creation, no? Journal of Developmental Entrepreneurship, 11(04), 319-343. 
Driver, M. (2011). An interview with Michael Porter: Social Entrepreneurship and the Transformation of Capitalism. Academy of Management Learning and Education, 11(3), 421-431.

https://doi.org/10.5465/amle.2011.0002

Eisenhardt, K. M. (1989). Building Theories from Case Study Research. The Academy of Management Review, 14(4), 532-550. https://doi.org/10.2307/258557

Estrin, S., Mickiewicz, T., \& Stephan, U. (2013). Entrepreneurship, Social Capital, and Institutions: Social and Commercial Entrepreneurship Across Nations. Entrepreneurship: Theory and Practice, 37(3), 479-504. https://doi.org/10.1111/etap.12019

Fajnzylber, P., Lederman, D., \& Loayza, N. (2002). Inequality and Violent Crime *. The Journal of Law \& Economics, 45(1), 1-39. https://doi.org/10.1086/338347

Fu, Y., Kok, R. A. W., Dankbaar, B., Ligthart, P. E. M., \& van Riel, A. C. R. (2018). Factors affecting sustainable process technology adoption: A systematic literature review. Journal of Cleaner Production, 205, 226-251.

Fukuyama, F. (2001). Social capital, civil society and development. Third World Quarterly, 22(1), 7-20. https://doi.org/10.1080/713701144

Gasparini, L., Cruces, G., \& Tornarolli, L. (2011). Recent Trends in Income Inequality in Latin America. Economía, 11(2), 147-190.

Giddens, A. (1979). Central problems in social theory : action, structure and contradiction in social analysis. London: London: Macmillan.

Giddens, A. (1984). The constitution of society : outline of the theory of structuration. Cambridge: Cambridge : Polity Press.

Glaeser, E. L., Resseger, M., \& Tobio, K. (2009). Inequality in Cities. Journal of Regional Science, 49(4), 617-646. https://doi.org/10.1111/j.1467-9787.2009.00627.x

Goyal, S., Sergi, B. S., \& Jaiswal, M. (2015). How to Design and Implement Social Business Models for Base-ofthe-Pyramid (BoP) Markets? European Journal of Development Research, 27(5), 850-867. https://doi.org/10.1057/ejdr.2014.71

Hall, J., \& Vredenburg, H. (2003). The Challenges of Innovating for Sustainable Development What to Read Next. Recuperado el 4 de Abril de 2020, de MIT Sloan Management Review website:

https://sloanreview.mit.edu/article/the-challenges-of-innovating-for-sustainable-development/

Hoskisson, R. E., Eden, L., Lau, C. M., \& Wright, M. (2000). Strategy in emerging economies. Academy of Management Journal, 43(3), 249-267.

London, T., \& Hart, S. L. (2011). Next generation business strategies for the base of the pyramid : new approaches for building mutual value. Upper Saddle River, N.J.: Upper Saddle River, N.J. : FT Press.

Lopez, J. H. (2006). A normal relationship?: poverty, growth, and inequality (L. Serven \& W. B. L. A. and the C. R. O. O. of the C. Economist, Eds.). Washington, D.C.: Washington, D.C. : World Bank, Latin America and the Caribbean Region, Office of the Chief Economist.

Lopez, J. H., \& Perry, G. (2008). Inequality in Latin America_ Determinants and Consequences (No. Policy Research Working Paper 4504). Recuperado de http://econ.worldbank.org.Theauthormaybecontactedathlopez@worldbank.org.

Maclean, M., Harvey, C., \& Gordon, J. (2013). Social innovation, social entrepreneurship and the practice of contemporary entrepreneurial philanthropy. International Small Business Journal, 31(7), 747-763. https://doi.org/10.1177/0266242612443376 
Mair, J., \& Marti, I. (2006). Social entrepreneurship research: A source of explanation, prediction, and delight. Journal of World Business, 41(1), 36-44.

Meyskens, M., Carsrud, A. L., \& Cardozo, R. N. (2010). The symbiosis of entities in the social engagement network: The role of social ventures. Entrepreneurship \& Regional Development, 22(5), 425-455. https://doi.org/10.1080/08985620903168299

Mongelli, L., \& Rullani, F. (2017). Inequality and marginalisation: social innovation, social entrepreneurship and business model innovation: The common thread of the DRUID Summer Conference 2015. Industry and Innovation, 24(5), 446-467. https://doi.org/10.1080/13662716.2017.1295365

Mulgan, G. (2006). The process of social innovation. Innovations: Technology, Governance, Globalization, 1(2), 145-162.

Naciones Unidas. (2020). Sustainable Development Goals .: Sustainable Development Knowledge Platform. Retrieved July 4, 2020, from https://sustainabledevelopment.un.org/?menu=1300

Nakata, C., \& Weidner, K. (2012). Enhancing product adoption at the BoP: A Contextualized Model. Journal of Product Innovation Management, 29(1), 21-32.

Nicholls, A. (2008). Social entrepreneurship : new models of sustainable social change. Oxford: Oxford : Oxford University Press.

Nielsen, C., \& Samia, P. M. (2008). Understanding key factors in social enterprise development of the BOP: a systems approach applied to case studies in the Philippines. The Journal of Consumer Marketing, 25(7), 446-454. https://doi.org/10.1108/07363760810915662

Núñez, G. P. (2010). Emprendimiento social: integrando a los excluidos en el ámbito rural. Revista de Ciencias Sociales (Ve), 16(4), 579-590.

Organización de las Naciones Unidas para la Educación, la Ciencia y la Cultura. (2014). Regional report about education for all in Latin America and the Caribbean. Recuperado de http://www.unesco.org/new/fileadmin/MULTIMEDIA/HQ/ED/ED_new/pdf/LAC-GEM-2014-ENG.pdf

Perry, G., Arias, O., López, H., Maloney, W., \& Servén, L. (2006). Poverty reduction and growth : virtuous and vicious circles. Washington, DC: World Bank.

Porter, M., \& Kramer, M. (2011). Creating Shared Value: How to Reinvent Capitalism- and Unleash a Wave of Innovation and Growth. Harvard Business Review, 49-58. https://doi.org/10.1108/09600039410055963

Prahalad, C. K. (2006). The fortune at the bottom of the pyramid. In Fortune at the bottom of the pyramid: eradicating poverty through profits (Paperback). Upper Saddle River, NJ : Wharton School Publishing.

Rangan, S., Samii, R., \& Van Wassenhove, L. N. (2006). Constructive Partnerships: When Alliances between Private Firms and Public Actors Can Enable Creative Strategies. The Academy of Management Review, 31(3), 738-751. https://doi.org/10.5465/AMR.2006.21318928

Salim, N., Ab Rahman, M. N., \& Abd Wahab, D. (2019). A systematic literature review of internal capabilities for enhancing eco-innovation performance of manufacturing firms. Journal of Cleaner Production, 209, 14451460.

Santos, F. (2012). A Positive Theory of Social Entrepreneurship. Journal of Business Ethics, 111(3), 335-351. https://doi.org/10.1007/s10551-012-1413-4

Santos, F., Pache, A. C., \& Birkholz, C. (2015). Making hybrids work: Aligning business models and organizational design for social enterprises. California Management Review, 57(3), 36-58.

https://doi.org/10.1525/cmr.2015.57.3.36 
Seelos, C. (2010). Theorizing And Strategizing With Models: Generative Models Of Business Models (No. 857; Vol. 5). Recuperado de http://ssrn.com/abstract $=1645200$

Seelos, C., \& Mair, J. (2005). Social entrepreneurship: Creating new business models to serve the poor. Business Horizons, 48(3), 241-246. https://doi.org/10.1016/j.bushor.2004.11.006

Sen, A. (2001). Development as freedom. Oxford, UK: Oxford University Press.

Smith, A. (2001). An Inquiry into the nature and causes of the wealth of nations (issuing body ProQuest (Firm), Ed.). London : Electric Book Co.

Spear, R. (2006). Social entrepreneurship: a different model? International Journal of Social Economics, 33(5/6), 399-410. https://doi.org/10.1108/03068290610660670

Székely, M., \& Mendoza, P. (2017). Declining inequality in Latin America: structural shift or temporary phenomenon? Oxford Development Studies, 45(2), 204-221. https://doi.org/10.1080/13600818.2016.1140134

Vennesson, P. (2008). Case studies and process tracing: Theories and practices. Teoksessa D. della Porta \& M. Keating (toim.) Approaches and methodologies in the social sciences: 223-240. In D. Della Porta \& M. Keating (Eds.), Approaches and Methodologies in the Social Sciences. A Pluralist Perspective (pp. 223-239). New York, NY, US: Cambridge University Press.

Yin, R. K. (2014). Case study research : design and methods (Fifth edit). Thousand Oaks, California : SAGE.

Yunus, M. (2008). Creating a World Without Poverty: Social Business and the Future of Capitalism. Global Urban Development, 4(2), 1-19. Recuperado de https://www.globalurban.org/GUDMag08Vol4lss2/Yunus.pdf

Yunus, M., Moingeon, B., \& Lehmann-Ortega, L. (2010). Building social business models: Lessons from the grameen experience. Long Range Planning, 43(2-3), 308-325. https://doi.org/10.1016/j.Irp.2009.12.00

Zahra, S. A., Gedajlovic, E., Neubaum, D. O., \& Shulman, J. M. (2009). A typology of social entrepreneurs: Motives, search processes and ethical challenges. Journal of Business Venturing, 24(5), 519-532. https://doi.org/10.1016/j.jbusvent.2008.04.007

Zahra, S. A., Rawhouser, H. N., Bhawe, N., Neubaum, D. O., \& Hayton, J. C. (2008). Globalization of social entrepreneurship opportunities. Strategic Entrepreneurship Journal, 2(2), 117-131.

https://doi.org/10.1002/sej.43

Esta obra está bajo una Licencia Creative Commons Attribución-NoCommercial 4.0 International

\section{(cc) BY-NC}

